\title{
Hard x-ray optics: from HEFT to NUSTAR
}

Jason E. Koglin, C. M. Hubert Chen, Jim C. Chonko, Finn E. Christensen, William W. Craig, et al.

Jason E. Koglin, C. M. Hubert Chen, Jim C. Chonko, Finn E. Christensen, William W. Craig, Todd R. Decker, Charles J. Hailey, Fiona A. Harrison, Carsten P. Jensen, Kristin K. Madsen, Michael J. Pivovaroff, Marcela Stern, David L. Windt, Eric Ziegler, "Hard x-ray optics: from HEFT to NuSTAR," Proc. SPIE 5488, UV and Gamma-Ray Space Telescope Systems, (11 October 2004); doi: 10.1117/12.552470

SPIE Event: SPIE Astronomical Telescopes + Instrumentation, 2004, Glasgow, United Kingdom 


\title{
Hard X-ray Optics: from HEFT to NuSTAR
}

\author{
Jason E. Koglin ${ }^{\mathrm{a}}$, C. M. Hubert Chen ${ }^{\mathrm{b}}$, Jim C. Chonko ${ }^{\mathrm{a}}$, Finn E. Christensen ${ }^{\mathrm{c}}$, William W. Craig ${ }^{\mathrm{d}}$, \\ Todd R. Decker ${ }^{d}$, Charles J. Hailey ${ }^{\mathrm{a}}$, Fiona A. Harrison ${ }^{\mathrm{b}}$, Carsten P. Jensen ${ }^{\mathrm{c}}$, Kristin K. Madsen ${ }^{\mathrm{c}}$, \\ Michael J. Pivovaroff ${ }^{d}$, Marcela Stern ${ }^{\mathrm{a}}$, David L. Windt ${ }^{\mathrm{a}}$, Eric Ziegler ${ }^{\mathrm{e}}$ \\ ${ }^{a}$ Columbia Astrophysics Laboratory, New York, NY \\ ${ }^{\mathrm{b}}$ California Institute of Technology, Pasadena, CA \\ ${ }^{c}$ Danish Space Research Institute, Copenhagen, Denmark \\ ${ }^{\mathrm{d}}$ Lawrence Livermore National Laboratory, Livermore, CA \\ ${ }^{\mathrm{e}}$ European Sychrotron Radiation Facility, Grenoble Cedex, France
}

\begin{abstract}
Focusing optics are now poised to dramatically improve the sensitivity and angular resolution at energies above $10 \mathrm{keV}$ to levels that were previously unachievable by the past generation of background limited collimated and coded-aperture instruments. Active balloon programs (HEFT), possible Explorer-class satellites (NuSTAR - currently under Phase A study), and major X-ray observatories (Con-X HXT) using focusing optics will play a major role in future observations of a wide range of objects including young supernova remnants, active galactic nuclei, and galaxy clusters. These instruments call for low cost, grazing incidence optics coated with depth-graded multilayer films that can be nested to achieve large collecting areas. Our approach to building such instruments is to mount segmented mirror shells with our novel error-compensating, monolithic assembly and alignment (EMAAL) procedure. This process involves constraining the mirror segments to successive layers of graphite rods that are precisely machined to the required conicapproximation Wolter-I geometry. We present results of our continued development of thermally formed glass substrates that have been used to build three HEFT telescopes and are proposed for NuSTAR. We demonstrate how our experience in manufacturing complete HEFT telescopes, as well as our experience developing higher performance prototype optics, will lead to the successful production of telescopes that meet the NuSTAR design goals.
\end{abstract}

KEYWORDS: Hard X-ray telescope, X-ray optics, segmented glass, thermally-slumped, HEFT, NuSTAR

\section{MOTIVATION FOR HARD X-RAY OPTICS DEVELOPMENT}

A new generation of hard X-ray instruments is required to pursue a variety of science objectives:

- Conduct a census for black holes on all scales, achieved through deep, wide-field surveys of extragalactic fields and the Galactic center.

- Map radioactive material in young supernova remnants to study the birth of the elements and to understand how stars explode.

- Explore relativistic jets of particles from the most extreme active galaxies to understand what powers giant cosmic accelerators.

- Study cosmic ray origins and the extreme physics around collapsed stars

We have developed thermally-formed glass substrates and a unique mounting technique to build the high performance, lightweight telescopes with large effective area to enable new discovery in the 6-80 keV energy band. Our approach is currently being demonstrated through the High Energy Focusing Telescope (HEFT), a balloon born mission slated to fly in the fall of 2004. HEFT will not only be among the first focusing telescopes to yield significant science returns as a balloon mission, it also promises to demonstrate key technologies that are proposed for future satellite missions. The thermally-formed glass and mounting approach developed over nine years for HEFT are being proposed for a small

\footnotetext{
*Correspondence: koglin@astro.columbia.edu, http://www.astro.columbia.edu/ koglin
} 
explorer class satellite, the Nuclear Spectroscopic Telescope Array (NuSTAR) ${ }^{1}$. The NuSTAR mission will be the first satellite instrument to employ focusing optics in the 6 to $80 \mathrm{keV}$ hard X-ray band.

Since the HEFT design and production process has been described in detail elsewhere ${ }^{2,3,4}$, we will begin in Section 2 by giving an overview of the NuSTAR optics design and production process, with particular attention given where the design differs from HEFT. Then in Section 3, production information and calibration results from the first three HEFT optics modules will be presented which demonstrate continued improvement in both image performance and overall production quality control. In Section 4, we will detail the substrate selection process that will enable us to boost the image performance for NUSTAR to 40 " from the current one arcminute results demonstrated for the third HEFT optics module. Finally, data from our optics development program are presented in Section 5, which demonstrate our ability to meet the sensitivity goals of NuSTAR both in terms of angular resolution and multilayer response.

\section{NUSTAR OPTICS DESIGN}

The NuSTAR instrument builds on the successful development of the HEFT balloon program, using a simple design with extensive heritage and experience. The optics design and proposed production process for NuSTAR is very similar to that used to successfully build three HEFT telescopes. This section will give an overview of the production process, emphasizing how it will be implemented in the context of the NuSTAR, which must be executed on a short timescale with the high quality assurance that is required for a SMEX satellite mission.

\subsection{Overall Telescope Design}

The NuSTAR instrument is an array of three coaligned hard X-ray telescopes, which together provide the unique sensitivity, sub-arcminute resolution, and few-arcsecond positioning required to achieve NuSTAR's science goals. Each telescope consists of a grazing incidence optics module that focuses onto a shielded solid-state pixel detector. The optics and detectors are separated by a mast, which extends the focal length to 10 meters after launch. The NuSTAR optics use thin, segmented glass shells that approximate a Wolter-I geometry. They are coated with depth-graded multi-layers (W/Si and $\mathrm{Pt} / \mathrm{SiC}$ ) to extend the bandpass and field of view beyond what can be achieved with simple metal surfaces. Three flight modules have been completed and characterized for HEFT, giving high confidence in the mirror fabrication approach, performance, and

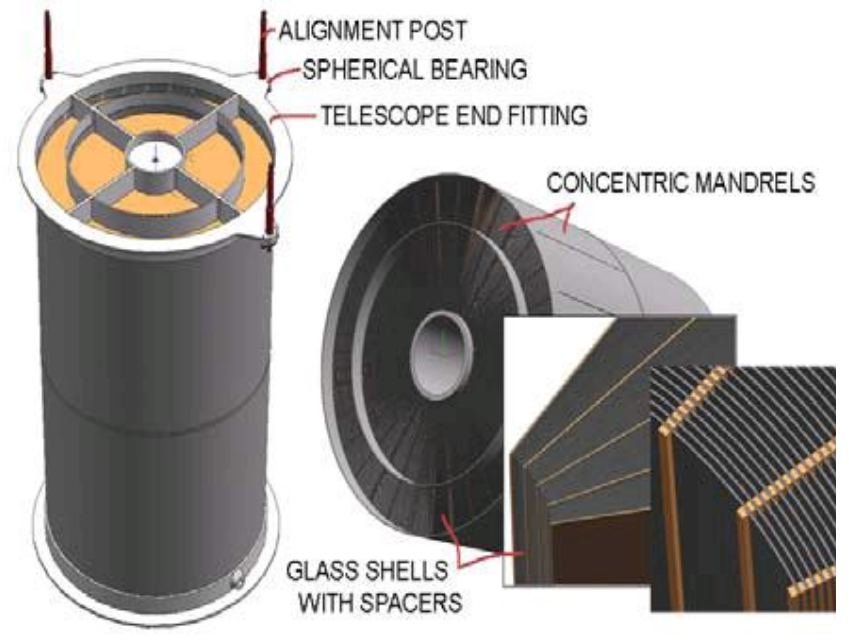

Figure 1: NuSTAR optics design. production schedule. The CdZnTe pixel detectors give high quantum efficiency and good spectral resolution with no need for cryogenic operation. Together with their associated electronics, they are housed in a lead and plastic scintillator shielding. The design is directly based on the HEFT focal plane, and in most cases no modifications are required, giving high confidence in the performance characteristics.

\subsection{Optics Mechanical Design}

The three optical modules on NuSTAR collect the incoming hard X-rays and focus them on to the detector. The optical design is a conical approximation to a Wolter-I, providing high performance across the 12' maximum field of view. The optics (c.f., Figure 1), are built of thermally formed glass substrates, precisely held in position by epoxy and graphite spacers. The entire assembly is built on, and aligned to, a central mandrel. After construction, an external can, to protect the optics during subsequent handling, and a support spider are attached. Alignment pins are added during optics calibration and used to tie the optical modules to the optical bench during instrument integration. Each telescope has two end fittings that provide handling and attachment features and carry loads between the inner, intermediate, and outer mandrels. During fabrication, glass and graphite layers are built outward from the central mandrel and all ground handling and alignment activities use the inner mandrel as a support and reference point. Once the telescopes are 
mounted to the telescope bench, each is supported and aligned from attachment points on the outer mandrel. The end fittings provide a structural link back to the center mandrel and distribute loading across the telescope end faces. The baseline optics design for NuSTAR, including the component masses, is detailed in Table 1.

\subsection{Manufacturing Formed Glass Substrates}

The substrates used to build the NuSTAR optics are made of thermally formed glass. The glass, Schott D-263, has been used successfully to produce the optics for the HEFT balloon program and is also used by GSFC in producing prototype optics for Constellation-X. The HEFT program has now produced more than 5000 flight mirror segments to build three HEFT telescopes. Including process development and prototyping, over 10,000 substrates have been produced. The NuSTAR substrate production is based on the procedures and lessons learned from HEFT, with upgrades in technology at high leverage locations in the manufacturing chain.

The D263 glass baselined for NuSTAR was developed for flat panel displays and is produced continuously in large volume at Schott's Desag glass works in Hanover, Germany. The flat glass, which is smooth and flat on all relevant length scales, is visually inspected for flaws. If it is deemed acceptable, then it is then positioned on a quartz mandrel inside a commercial oven, with a window and ceramic fixturing to support the glass forming process. The glass is heated so that it 'slumps' into the mandrel, forming a curved section close in radius to the final desired conical segment.

A laser scanning system is used to measure the performance of the thermally formed glass. ${ }^{5}$ The system uses a laser beam reflection from the front surface of the glass, and a position sensitive detector, to measure the glass surface profile within a few arcseconds. The data from each scan is automatically analyzed to produce a $3 \mathrm{D}$ surface map. The glass is then "virtually mounted" and the results entered into a database. The best performing portion of the substrate is selected through a process that is explained in more detail in Section 4. The substrates that meet the NuSTAR performance criteria are passed to the cutting technicians to be cut to the final shape. A scribe and break method was used to cut the glass for the first three HEFT optics. This has proven to be an effective method, and over 5000 pieces have been cut with a yield of $92 \%$. In order to improve the cut quality assurance, NuSTAR will use a hot wire instead of a scribe to cut the glass. This hot wire procedure, which was developed at GSFC and has been successfully prototyped for HEFT, produces a cleaner cut and reduces the chance of fracture during subsequent handling.

\begin{tabular}{|l|r|l|}
\hline Modules & 3 & \\
\hline Focal Length & 10037 & $\mathrm{~mm}$ \\
\hline Glass Thickness & 200 & $\mu \mathrm{m}$ \\
\hline Radius Min & 54.9 & $\mathrm{~mm}$ \\
\hline Radius Max & 168.6 & $\mathrm{~mm}$ \\
\hline Shell Length & 200 & $\mathrm{~mm}$ \\
\hline Glass Density & 2.51 & $\mathrm{~g} / \mathrm{cm}^{3}$ \\
\hline Detector Size (side) & 25 & $\mathrm{~mm}$ \\
\hline Field of View (min - max) & 12.0 & $\mathrm{arcmin}$ \\
\hline Layers per module & 130 & \\
\hline Glass Mass & 16.0 & $\mathrm{~kg}$ \\
\hline Graphite Mass & 1.9 & $\mathrm{~kg}$ \\
\hline Epoxy Mass & 1.4 & $\mathrm{~kg}$ \\
\hline Multilayer Mass & 0.3 & $\mathrm{~kg}$ \\
\hline Mandrel \& Mounting Hardware & 4.9 & $\mathrm{~kg}$ \\
\hline Total Optic & 24.5 & $\mathrm{Kg}$ \\
\hline
\end{tabular}

Table 1: Optical design parameters and component masses.

The 130 layers of a NuSTAR optic module each consist of 32 substrates. For NuSTAR, three flight optics and one spare optic require 16,640 substrates. Based on the experience of HEFT, a combined yield for glass selection, handling and cutting is expected to be $\sim 50 \%$. Thus approximately 35,000 substrates must be manufactured. Twenty-four ovens will be used for NuSTAR, as compared to the 12 ovens currently used for HEFT. With a production capability of over 20 substrates per week per oven based on experience with HEFT, the requirement for NuSTAR can be achieved in less than 18 months.

\subsection{Depth-Graded Multilayers}

Depth-graded multilayers are applied to the optical surfaces in a production coating facility at DSRI that uses planar magnetron sputtering. The specific multilayer design depends on graze angle, which scales with the optic radius. The 130 shells are divided into 20 groups optimized for a particular range of graze angles. Before coating, the substrates are cleaned using a three step ultrasonic process and sorted by radius for application of the appropriate design. The DSRI facility can coat $0.8 \mathrm{~m}^{2}$ in substrate surface area in a single run (pump-down, coating and venting of the chamber); each run is a one-day operation. Producing the required number of substrates consistent with the NuSTAR schedule requires 
coating $1.9 \mathrm{~m}^{2} /$ week, compared to the $4 \mathrm{~m}^{2} /$ week capability, providing significant margin. An additional facility at Columbia provides backup and coating capability for prototypes and engineering models. Quality is assured for each coating run by including a silicon wafer as a witness that is characterized at $8 \mathrm{keV}$ for reflectance spectra. These reflectance data, as well as housekeeping data from the coating chamber are automatically logged to the optics database, also updated to reflect the coating applied to each substrate. The material combinations for the coatings depend on graze angle - the inner 14 radius groups (about 2/3 of the geometric area) are coated with $\mathrm{Pt} / \mathrm{SiC}$, and the remaining are coated with $\mathrm{W} / \mathrm{SiC}$.

\subsection{Substrate Mounting}

The coated substrates are assembled into an optic at an existing facility, developed for HEFT at Colorado Precision Products Incorporated (CPPI) ${ }^{*}$ in Boulder, CO. Our unique error-compensating, monolithic assembly and alignment (EMAAL) procedure involves constraining the mirror segments to precisely machined graphite spacers that run along the optical axis. In this process, the nominally cylindrical glass segments are forced to a conical form, and in the process, radial mismatches and some small twists in the glass are removed. In order to achieve large effective area, concentric layers of glass are stacked on top of each other starting with a central mandrel. Graphite spacers are first epoxied to the mandrel and then precisely machined to the correct radius and angle. Next, a layer of glass and second layer of spacers are epoxied to the first set of spacers. These spacers are then machined to the appropriate radius and angle. This process is repeated until the requisite number of layers is assembled.

A key point of this process is that each layer of spacers is machined with respect to the optic axis and not the last layer of glass. In this way, there is never any stack-up error during the telescope fabrication. Each layer requires one workday to complete. Two machines operating in parallel produce two layers/day, so that four NuSTAR optics modules can be assembled in just over one year.

\section{HEFT TELESCOPE PRODUCTION AND CALIBRATION RESULTS}

Production of the first HEFT telescope HF1 began in May 2002 and was completed nine months later. Assembly of HF1 began using three spacers per quint section for the first 22 layers. At this point, a switch to five spacers per quint section was made. In order to make this change, an intermediate mandrel was added for structural support from which to build the subsequent layers. The second HEFT flight module HF2, which was begun immediately after HF1 was completed, was assembled in a similar fashion over the next six months. For the third HEFT optic module, HF3, the innermost 12 layers were abandoned and the entire optic was built using five spacers over the course of the next five months. Both HF2 and HF3 were assembled at an average rate of $\sim 3.5$ layers per week. These three HEFT optic modules are pictured in Figure 2.

Both $8 \mathrm{keV}$ and high energy (18-68 keV) X-ray calibration data have been previously reported for HF1 in addition to LVDT metrology. ${ }^{3}$ Each independent measurement yielded consistent results, and the HPD performance of the complete optic was reported to be $1.3 \pm 0.1^{\prime}$ at $40 \mathrm{keV}$ with the inner shells having $1.6 \pm 0.1^{\prime}$ ' performance, the middle shells $1.1 \pm 0.1^{\prime}$, and the outer shells $1.4 \pm 0.1^{\prime}$ '. A clear improvement in performance was measured after changing from three to five spacers per quint section. The pre-mounted, free-standing mirrors are only nominally cylindrical with small radial mismatches and twists. The improvement in performance with greater spacer density results from the greater ability to remove out-of-phase roundness errors in the mirrors (i.e., twists such that the nominal graze angle in the mirror changes with azimuth angle). The goal of the EMAAL mounting method is not to improve the axial figure of the mirrors - the goal is to simply constrain the mirror to the correct radius and angle at the point of the graphite spacers. Away from the spacers, the intrinsic roundness errors in the mirrors will cause the nominal graze angle of the mirror to deviate slightly from the required graze. By increasing the spacer density, this type of error can be minimized.

This improvement with spacer density is also apparent in the $8 \mathrm{keV}$ X-ray metrology data for HF2, which was built in a similar manner to HF1. The performances of each individual X-ray pencil beam measurement, performed every 2.5 degrees for each layer, are detailed in the histogram plotted in Figure 3. The performances for quints with three and five spacers are plotted separately and clearly illustrate the improved performance that arises from using five spacers per quint section. The performance histogram for five spacer quints peaks around 60" with a broad tail toward higher HPD performance.

\footnotetext{
* http://www.coloradoprecision.com/
} 

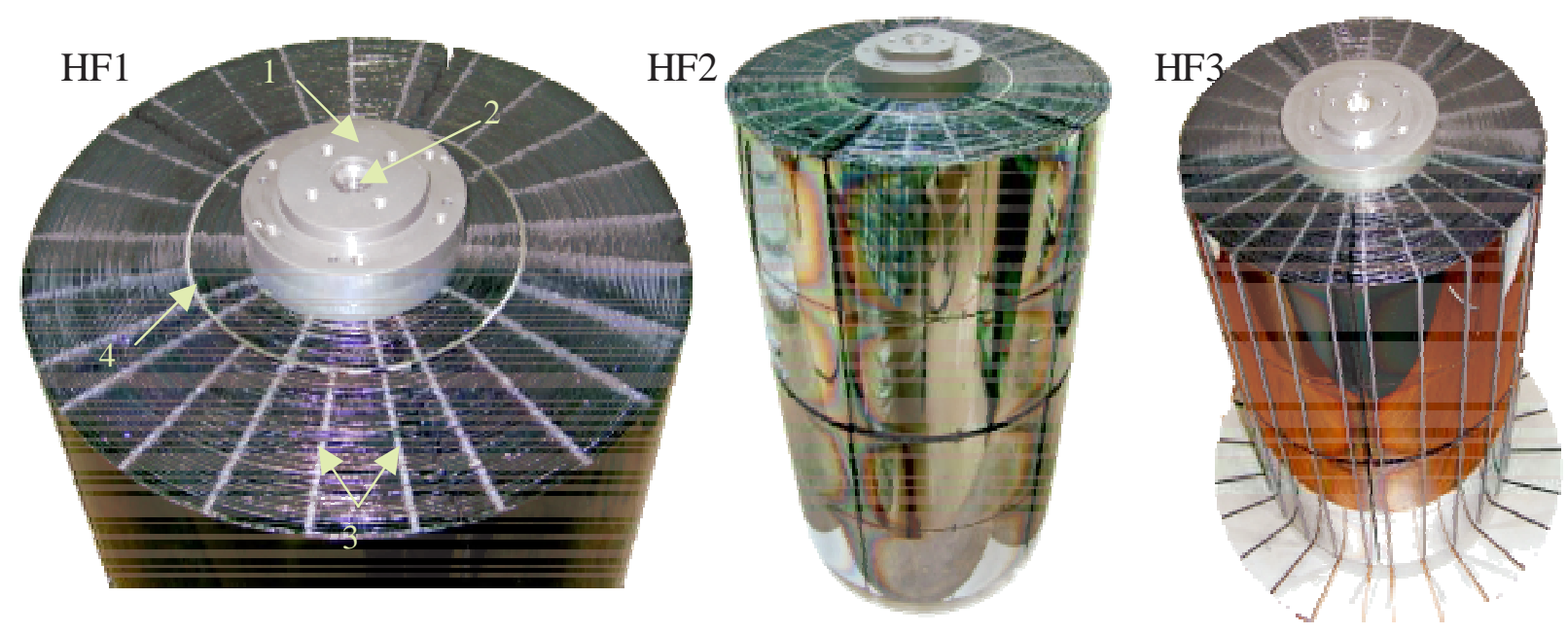

Figure 2: Three HEFT optic modules: HF1, HF2 and HF3. The following details are noted for HF1: 1 - the central titanium mandrel; 2 - precision alignment cone that defines the optical axis; 3 - stacks of graphite spacers; 4 - intermediate mandrel used for structural support when switching between 3 and 5 spacers per quint section.

Through continued improvement in both substrate production and mounting process control, we are demonstrating more uniform mounted mirror performance. This is illustrated in Figure 4, which shows the individual LVDT performance versus layer for HF3. Note that with the exception of a set of two mirrors in layer 65 where a rare mounting error presumably occurred, the mirrors in the last half of the telescope perform consistently near 50" HPD with relatively little spread in performance. Analysis of this LVDT data yields a performance of 57" HPD for the entire HF3 telescope.

A Histogram of the LVDT performance measurements for the three HEFT optics modules is plotted in Figure 5. A clear trend toward an improved performance from HF1 to HF3 is evident in this plot. In addition to improved performance, we have demonstrated a reliable manufacturing process in which mirrors are slumped, coated and mounted at an average rate of 3.5 telescope layers per week.

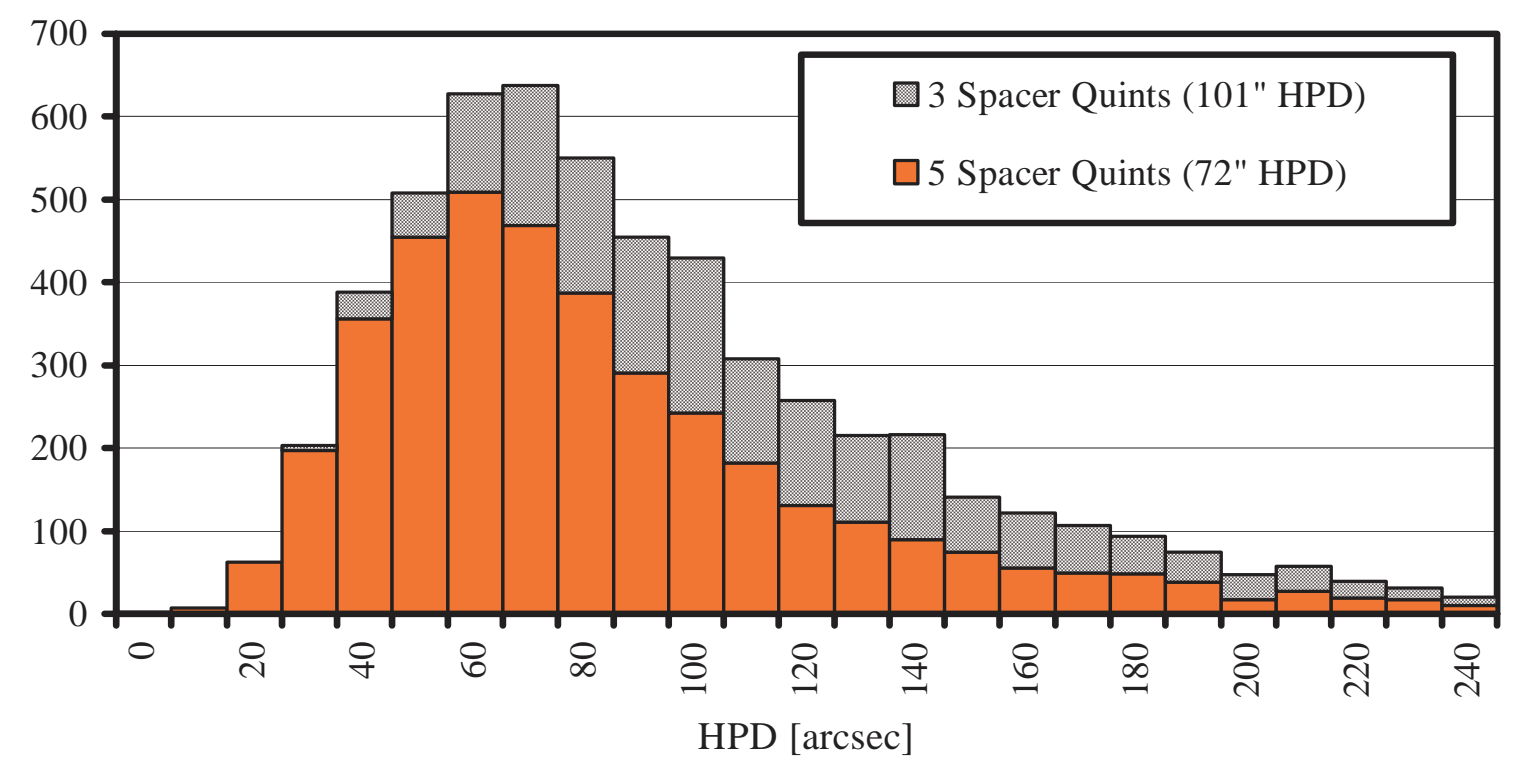

Figure 3: Histogram of HF2 X-ray pencil beam data measurements that were performed every 2.5 degrees over the entire optic. The performances for quints with three and five spacers are plotted separately and clearly illustrate the improved performance that arises from using five spacers per quint section. 


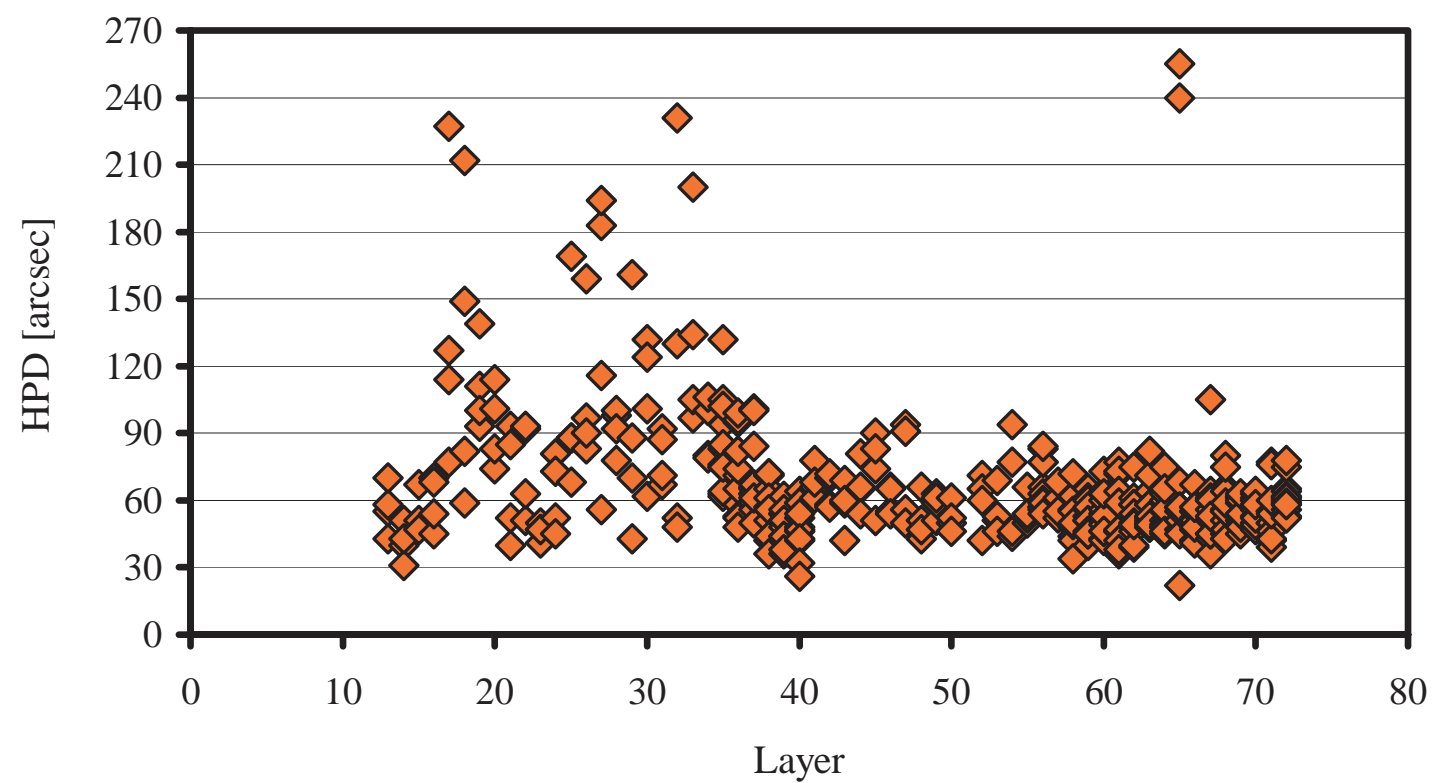

Figure 4: HF3 LVDT performance measurements plotted versus optic layer.

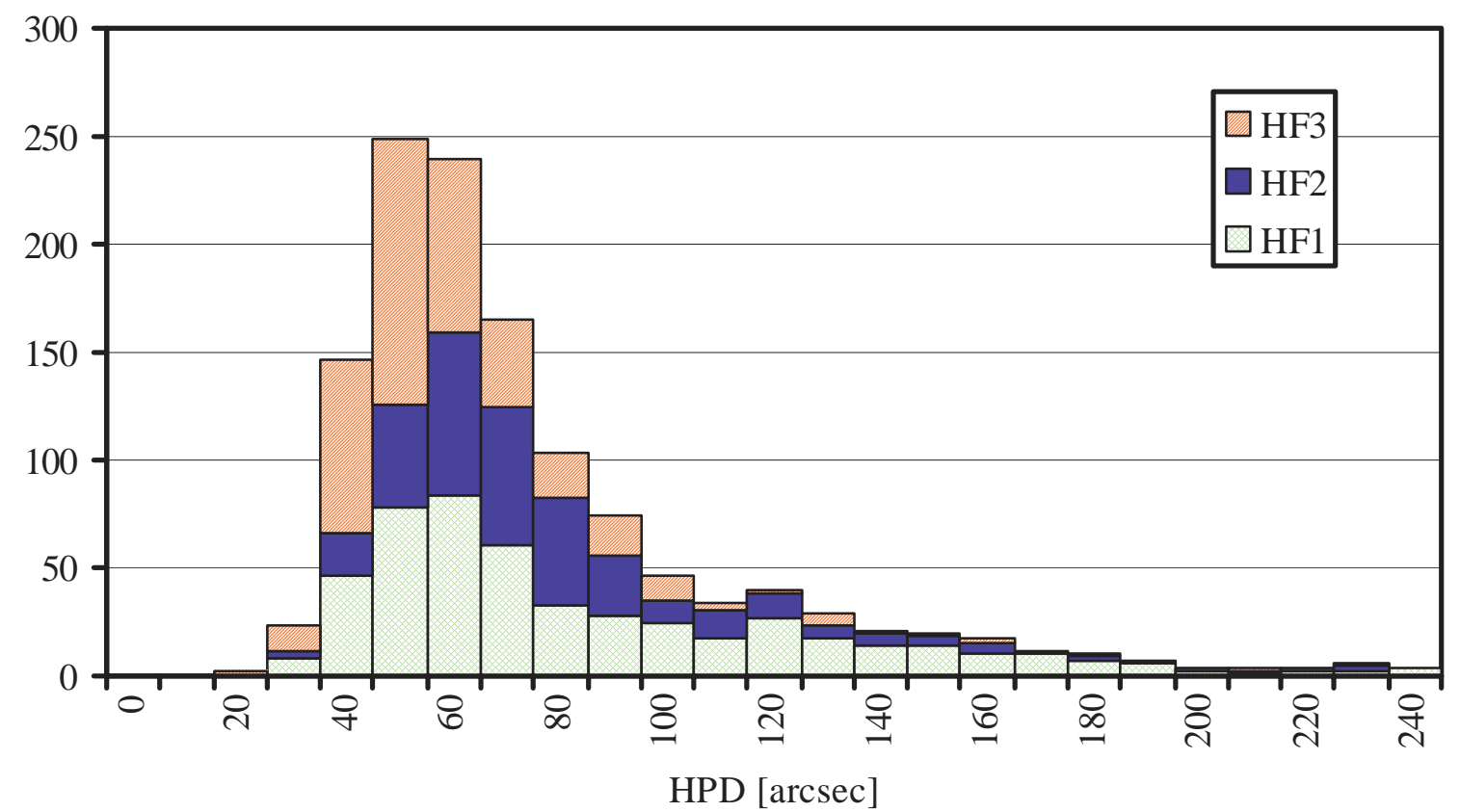

Figure 5: LVDT performance histogram for HF1, HF2 \& HF3. Note the continued improvement in glass/mounting performance.

\section{SUBSTRATE SELECTION}

Parallel to our efforts to refine our production processes for assembling flight grade telescopes for HEFT, we have been actively investigating the steps necessary to improve the angular resolution of our segmented glass optics for future satellite missions including NuSTAR. Key to this effort is performing detailed metrology of the glass substrates before they are mounted. It has been, and will continue to be, impractical to perform and analyze detailed metrology on every 
substrate produced for the HEFT balloon born mission. In spite of this, our method of sampling for quality assurance has proven to be quite effective, and we have converged to the one-arcminute goal we set for HEFT.

By performing full metrology on every substrate and using a more stringent acceptance criteria, we have previously demonstrated 45 " resolution in a prototype optic build at the central radius $(80 \mathrm{~mm})$ for HEFT. ${ }^{6}$ The substrates chosen for this prototype were from the same ensemble used to build the HEFT flight telescopes. The difference is that with this prototype, we were more effectively able to reject poorer performing shells that would have otherwise degraded the overall performance. Approximately half of the substrates available were selected through detailed laser metrology of every substrate in order to achieve this 45 " performance. This is in contrast to HEFT where sample metrology is performed only periodically for quality assurance of the general slumping parameter settings and nearly every substrate is actually accepted - a majority of which with no knowledge of the actual freestanding performance.

From this experience, we have developed a two-step selection process:

1. First Analysis Step:

a) Scan every substrate with axial scans every 10 degrees,

b) Analyze the central $10 \mathrm{~cm}$ of the entire quint section,

c) Select substrates with $<90$ " performance for Second analysis step.

2. Second Analysis Step:

a) Rescan substrates in detail every 2 degrees,

b) Analyze left, center and right octant $\left(45^{\circ}\right)$ sections,

c) Select best octant section with $<50^{\prime \prime}$ performance for coating and mounting.

This process is illustrated in Figure 6 for the HEFT inventory of $300 \mu \mathrm{m}$ glass substrates. This includes all 3568 substrates that have been scanned for HEFT, most of which were used in building one of the three HEFT telescopes or other prototype optic modules. The analysis steps outlined above have been applied to this archived data. Applying the first analysis step yields a combined performance of 75". This is consistent with the performance we have obtained from the three HEFT optic modules where essentially no substrate selection was performed. After selecting only those substrates with quint performance less than 90", the second analysis step was applied to select the best octant $\left(45^{\circ}\right)$ sections. The 2088 substrates that satisfy this condition (59\% of the original 3568 substrates) combine for a performance of 45". This is the performance achieved in the previously mentioned prototype built from this set of substrates using a similar selection yield. By performing an even more stringent acceptance criteria and only using substrates with less than 50" HPD, a telescope with 35" performance is expected. This selection process would then satisfy the NuSTAR performance goal of 40". Even with much less stringent selection criteria, the sub-arcminute goal of NuSTAR can be satisfied.

To meet the more stringent weight requirements of the NuSTAR satellite mission, $200 \mu \mathrm{m}$ thin glass substrates will be used. Through prototype development work, we have demonstrated that the performance of these thinner substrates is as good or perhaps even superior to the $300 \mu \mathrm{m}$ substrates we have used in building three flight telescopes. Equally important, we have demonstrated that it is not significantly more difficult to handle the thinner glass substrates. For example: our yield for cutting over 5000 slumped pieces of $300 \mu \mathrm{m}$ glass is $92 \%$ while it is $89 \%$ for nearly 200 slumped pieces of $200 \mu \mathrm{m}$ glass.

The same substrate selection process previously described has been applied to our inventory of 468 pieces of $200 \mu \mathrm{m}$ glass and is illustrated in Figure 7. The performance of these substrates is better than the $300 \mu \mathrm{m}$ glass previously plotted in Figure 6. This improved performance does not necessarily arise from the glass being intrinsically easier to slump; instead it simply reflects our improved production process controls. Indeed the $300 \mu \mathrm{m}$ substrates formed in the last half year during which time the $200 \mu \mathrm{m}$ substrates have been produced show a similar performance distribution. With no selection, we would expect to build a 65 " optic using these $200 \mu \mathrm{m}$ substrates in a quint configuration similar to the HEFT design (c.f., ${ }^{\text {st }}$ analysis step shown in Figure 7). After choosing only those quint substrates with performance less than 90" (72\% of the substrates) for the second analysis step, an optic with 37 " performance could be built by choosing the best octant section of each substrate. By further selecting only those octant substrates with less than 50" performance, an optic with 33" performance could be built. Using these selection criteria, the 40" goal for NuSTAR will be reached. 


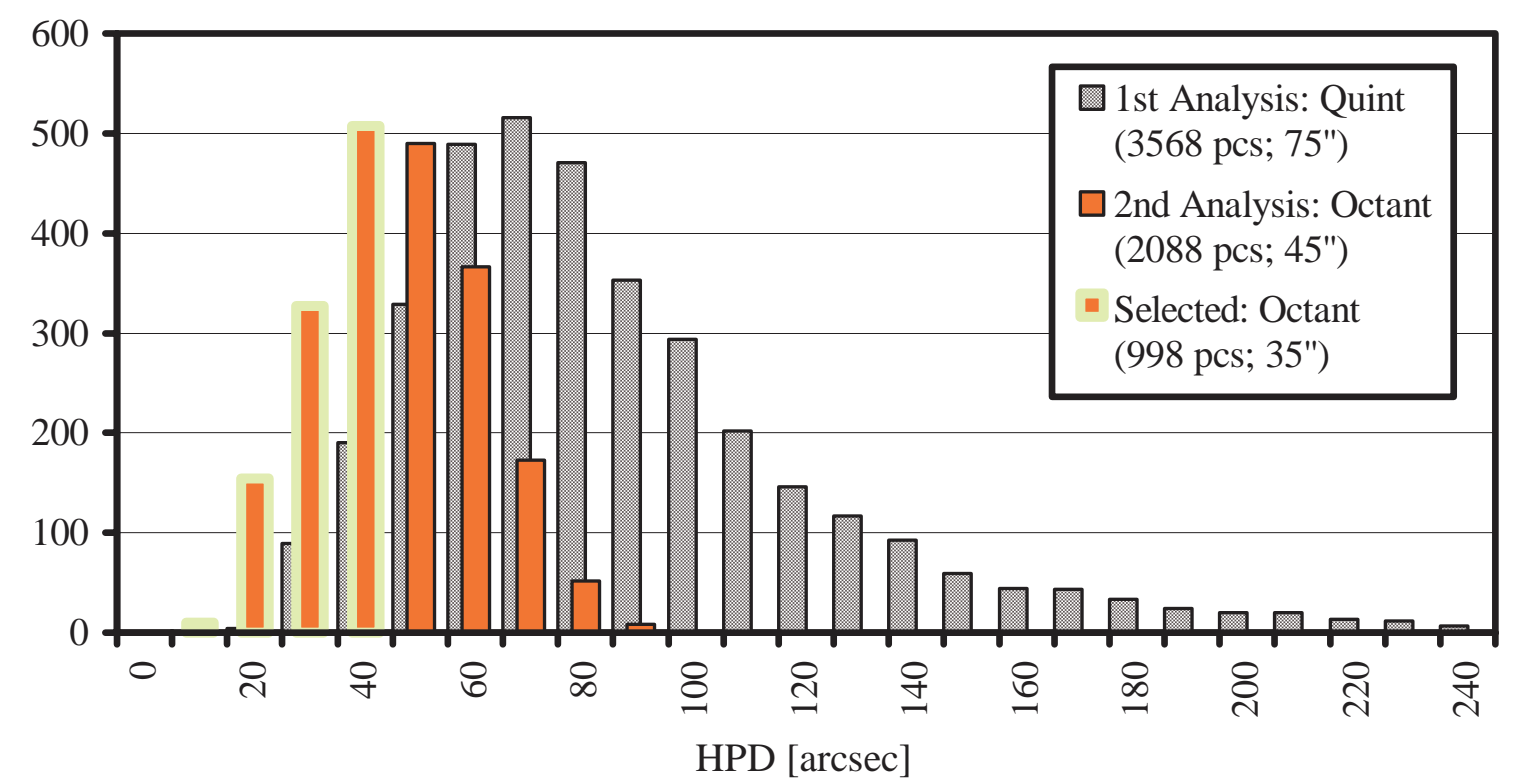

Figure 6: Performance histogram of entire inventory of 300 um glass segments highlighting the two-step substrate selection process. The number of substrates and predicted HPD performance is listed for the first analysis step in which all substrates are analyzed as 10 $\mathrm{cm}$ quint sections. Those substrates with performance less than 90 " are selected for the second analysis step in which the best octant sections is chosen. Finally only those quint sections with performance less than 50" are selected for mounting.

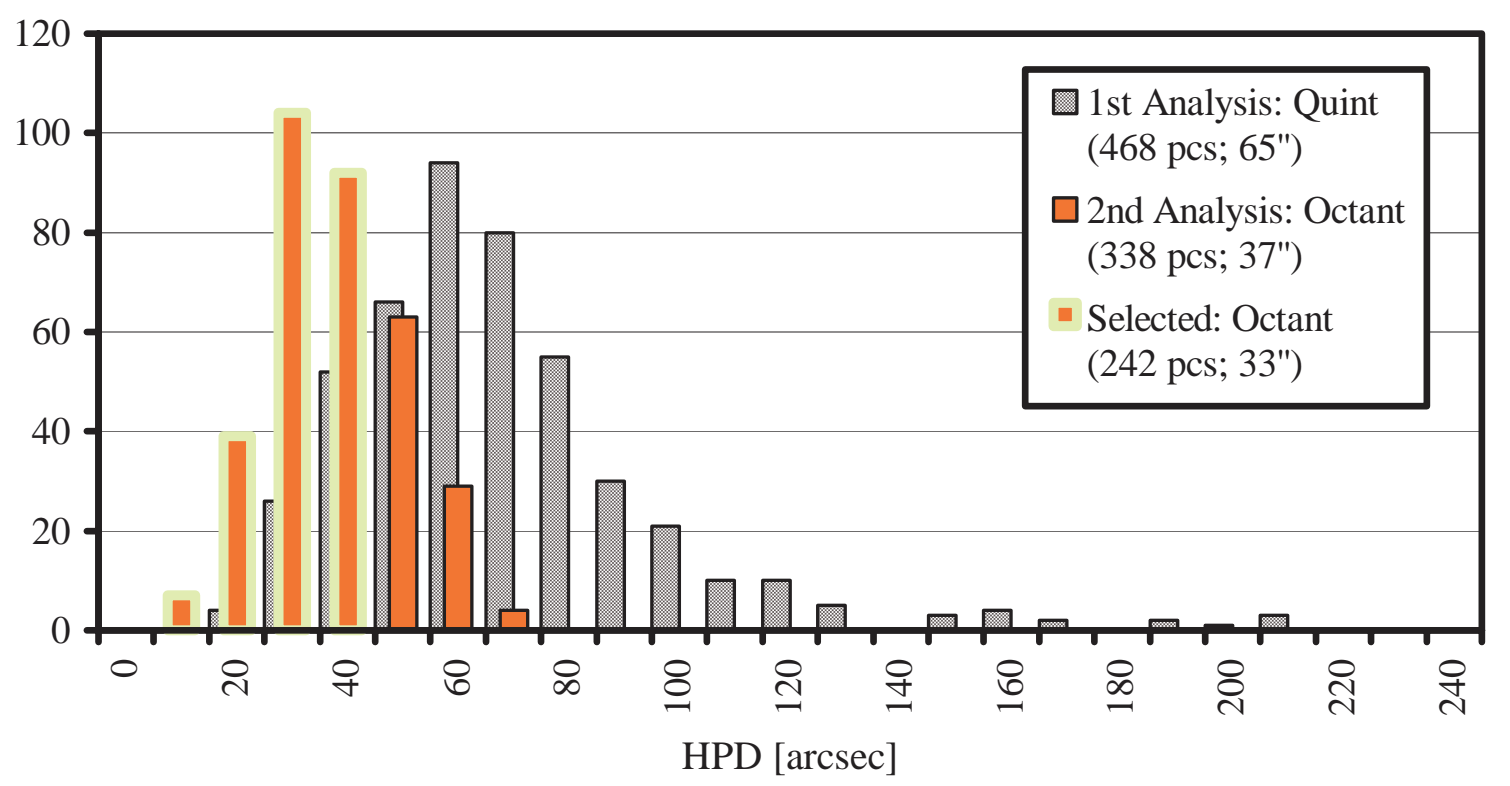

Figure 7: Performance histogram of entire inventory of 200 um glass similar to Figure 6.

\section{OPTICS DEVELOPMENT}

We have continued to build prototype optic modules both to confirm the predicted performance of our glass selection method and to test advanced mounting geometries and methods that either save time or reduce structural obscuration (e.g., use thinner graphite spacers). A recent prototype optic was built from the normal HEFT inventory of $300 \mu \mathrm{m}$ 
substrates selected according to the previously described selection method. A full layer, comprised of 32 octant mirrors coated with $\mathrm{W} / \mathrm{SiC}$ multilayers, was mounted at a nominal radius of $115 \mathrm{~mm}$ in a conic approximation Wolter-I configuration with a $6 \mathrm{~m}$ focal length (similar to the HEFT geometry). Each octant substrate, $10 \mathrm{~cm}$ long and $\sim 44^{\circ}$ in azimuth extent, was mounted on five graphite spacers that were each $0.75 \mathrm{~mm}$ thick and spaced every $10^{\circ}(\sim 20 \mathrm{~mm}$ in azimuth arclength). This is the mirror and spacer configuration proposed for NuSTAR.

\subsection{Angular resolution measurements}

High-resolution X-ray measurements at $8.048 \mathrm{keV}$ were performed every degree at the DSRI X-ray calibration facility. A triple-axis diffractometer configuration utilized high-resolution, perfect channel-cut monochromator and analyzer crystals - both $\mathrm{Si}(220)$ - in a non-dispersive configuration. The optic was first aligned optically so that it rotates about its axis with no visible wobble in precisely aligned pinholes at each end of the optic module that define the optical axis. The X-ray beam itself was then used to align the optic every $~ 30$ degrees. To perform the alignment, the optic was rotated in the horizontal plane to determine the position of maximum X-ray intensity passing through the pinholes at the front and back ends of the optic. In this way, residual wobble from the mechanical alignment of the optic was removed. It is important to emphasize that a Wolter-I optic is an imaging instrument. In this way, misalignment of the optic will not cause a displacement in the resulting image. The only consequence of any optic misalignment is that the measurements will be performed at a changing off-axis angle. The optic performance is constant up to several arcminutes off axis and only the throughput will be slightly degraded if the optic is slightly misaligned. The alignment of the optic is in fact estimated to be better than 15", and thus, it will have an insignificant effect on the resulting HPD measurements and the throughput will be degraded at most by a few percent.

To perform the scattering measurements, the optic is translated into the X-ray beam, and the analyzer crystal is rotated to probe the angle of the scattered radiation. In this way, the conic approximation error inherent in the optic design is not measured. The analyzer crystal accepts 5" as a nearly perfect step function. By scanning the analyzer crystal, a histogram of the reflected X-rays in angle space is recorded. Due to the excellent crystal resolution, essentially no background exists in this measurement and only small systematic uncertainties ( 5") are associated with co-adding the individual scans. This metrology method is thus very simple to analyze and provides a very accurate composite two-bounce image of the upper and lower shells at multiple azimuth positions. The same equipment and measurement procedure was performed using $40 \mathrm{keV} \mathrm{X}$-rays in the BM05 beamline at the European Synchrotron Research Facility (ESRF).

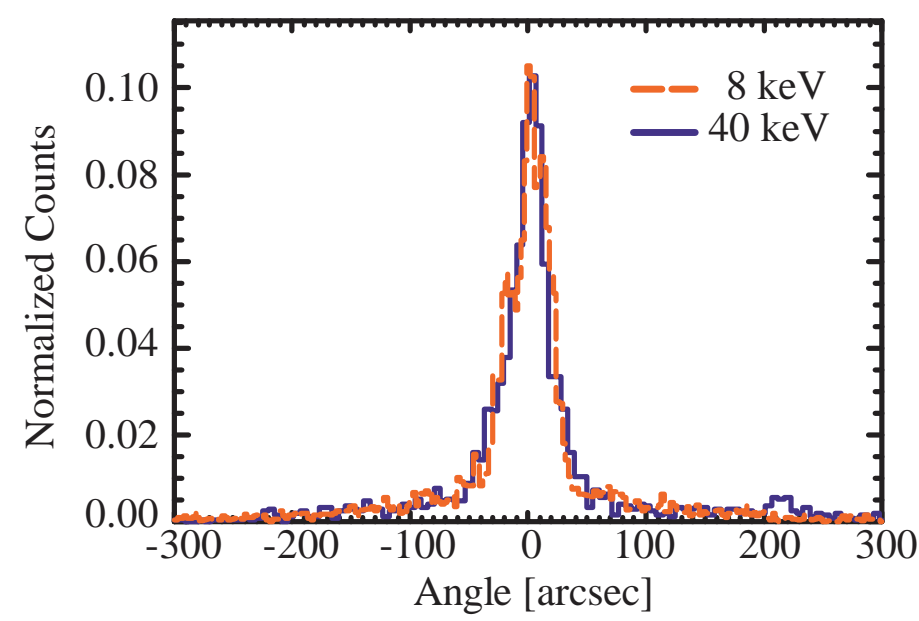

Figure 8: Typical example of X-ray pencil beam scans compared at 8 and $40 \mathrm{keV}$. These two measurements are consistent and yield a performance of 34" HPD.

The X-ray measurements at 8 and $40 \mathrm{keV}$ yield consistent results as illustrated in Figure 8. The 360 individual scans for each measurement range in performance from 15-65". The octant sectors have performances that range from 35-45" and combine for a total performance of $40 \pm 5$ ", where the error represents the estimated systematic uncertainty in the measurement. Due to the high statistics involved in these measurements, the statistical error is less than 1". A twodimensional image has been generated by combining the individual X-ray scans and is plotted in Figure 9. As was previously noted, the X-ray pencil beam measurements are performed in angle space and thus do not include the conicapproximation error intrinsic to our telescope design. The graze angle $\alpha$ for this prototype is $4.8 \mathrm{mrad}$ (greater than the outermost graze angle that will actually be used for NuSTAR). The conic approximation HPD error is given by

$$
\mathrm{HPD}_{\text {conic }}=(\alpha \mathrm{L}) /(2 \mathrm{~F}) \text {, }
$$

where $\mathrm{L}=200 \mathrm{~mm}$ is the length of the upper and lower mirror shells, and $\mathrm{F}=5896 \mathrm{~mm}$ is the focal distance of the telescope measured from the middle of the lower mirror shell to the focal plane. Thus, the conic approximation error for 
this prototype optic is 16 ". Given a Gauss distribution of slope defects, the conic approximation contribution to the overall HPD can be approximated as

$$
\mathrm{HPD}_{\text {total }}^{2} \cong \mathrm{HPD}_{\text {axial }}^{2}+2 \mathrm{HPD}_{\text {conic }}^{2} \text {. }
$$

This approximation works well for $\mathrm{HPD}_{\text {axial }}>2.5$ $\mathrm{HPD}_{\text {conic. }}$ In this way, the total HPD of this prototype optic is actually 46". However, the NuSTAR design calls for a focal length of $10,037 \mathrm{~mm}$ with a maximum graze angle of 4.16 mrad. Thus, the maximum conic-approximation error for NuSTAR will be limited to 8.5”. For a similar optic with 40.0" axial HPD performance, the conic-approximation will minimally increase the total HPD to only 41.7" for the outermost radius shell. The innermost radius NuSTAR shell has a graze angle of $1.35 \mathrm{mrad}$, which results in a conic-approximation error of only 2.8" and negligibly affects the total HPD for a 40 " optic. On average, the conic-approximation error will add only 1" to the axial HPD performance determined from X-ray pencil beam measurements for NuSTAR optics.

\section{$5.2 \mathrm{~W} / \mathrm{SiC}$ multilayer reflectivity}

The telescope effective area $A_{\text {eff }}$ is a product of several factors: the physical area of the telescope $\mathrm{A}_{\text {physical }}$; the multilayer reflectivity $\mathrm{R}(\mathrm{E}, \sigma, \alpha)$ which depends on the X-ray energy $E$, the surface roughness $\sigma$ and the graze angle $\alpha$; the physical obscuration $\varepsilon_{\text {obscuration }}$ due to the graphite

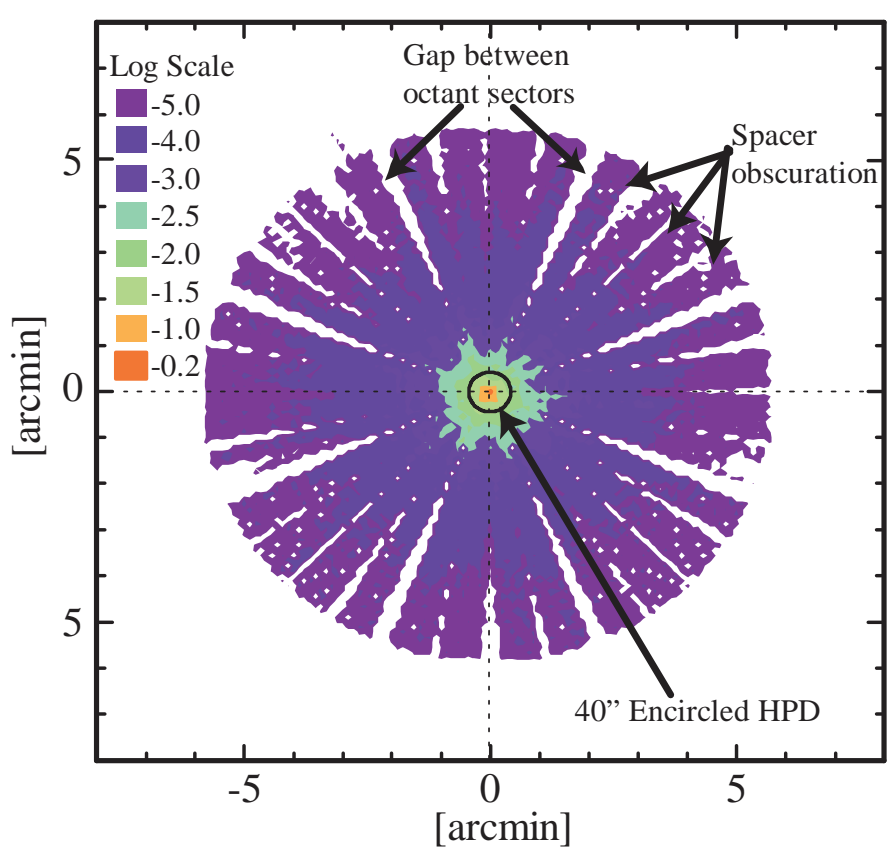

Figure 9: $8 \mathrm{keV} \mathrm{X}$-ray image generated by combining the individual pencil beam scan measurements. The detector field of view (FOV) for NuSTAR will be 8.2 ' with 10 " pixels. The central black circle denotes the 40" HPD. Obscuration due to spacers and gaps between octant sectors are indicated. Note the log intensity scale.

spacers and gaps between octant sectors where structural supports will be located; and axial throughput $\varepsilon_{\text {figure }}$ which can be attributed to figure errors in the mirrors. The effective area can be written as

$$
\mathrm{A}_{\text {eff }}=\mathrm{A}_{\text {physical }} \mathrm{R}(\mathrm{E}, \sigma, \alpha) \varepsilon_{\text {obscuration }} \varepsilon_{\text {figure. }} .
$$

For on axis sources the physical collection area is

$$
A_{\text {physical }}=2 \pi r_{\text {uо }} \alpha \mathrm{L} \text {. }
$$

where $\mathrm{r}_{\text {ио }}$ is the nominal radius of the upper mirror and $\mathrm{L}$ is the length of each upper and lower mirror shell.

Each NuSTAR optic module will be mounted on a support structure that will cover the gaps between octant sections. The eight supports will each be $\mathrm{w}_{\text {gap }}=2 \mathrm{~mm}$ wide - about the same width as the gap between octant sections. While the spacers themselves are only $0.75 \mathrm{~mm}$ wide, a small amount of epoxy excess around the spacer will cause added obscuration for each spacer. On average, each spacer obscures $\mathrm{w}_{\text {spacer }} \cong 1.0 \mathrm{~mm}$ of the segment. Thus, the total obscuration will be

$$
\varepsilon_{\text {obscuration }}=\left(\mathrm{n}_{\text {spacers }} \mathrm{w}_{\text {spacer }}+\mathrm{n}_{\text {segments }} \mathrm{w}_{\text {gap }}\right) /\left(2 \pi \mathrm{r}_{\text {uo }}\right),
$$

where $n_{\text {segments }}=8$ is the number of mirror segments and $n_{\text {spacers }}=40$ is the total number of spacers $(5$ for each octant section). The total obscuration for this prototype is $8 \%$, but it will range from $5 \%$ to $16 \%$ for the outermost to innermost NuSTAR radius shells, respectively.

The product of the reflectivity and axial throughput due to figure errors is measured directly using X-ray scattering measurements in a double-axis diffractometer configuration without the analyzer crystal in place. These measurements were performed using both 8 and $40 \mathrm{keV} \mathrm{X-rays} \mathrm{at} \mathrm{DSRI} \mathrm{and} \mathrm{ESRF,} \mathrm{respectively.} \mathrm{The} \mathrm{X-ray} \mathrm{flux} \mathrm{was} \mathrm{measured} \mathrm{with} \mathrm{a}$ pin diode detector and calibrated with the direct beam to give the measured throughput $\mathrm{T}_{\text {measured. }}$ As with the 


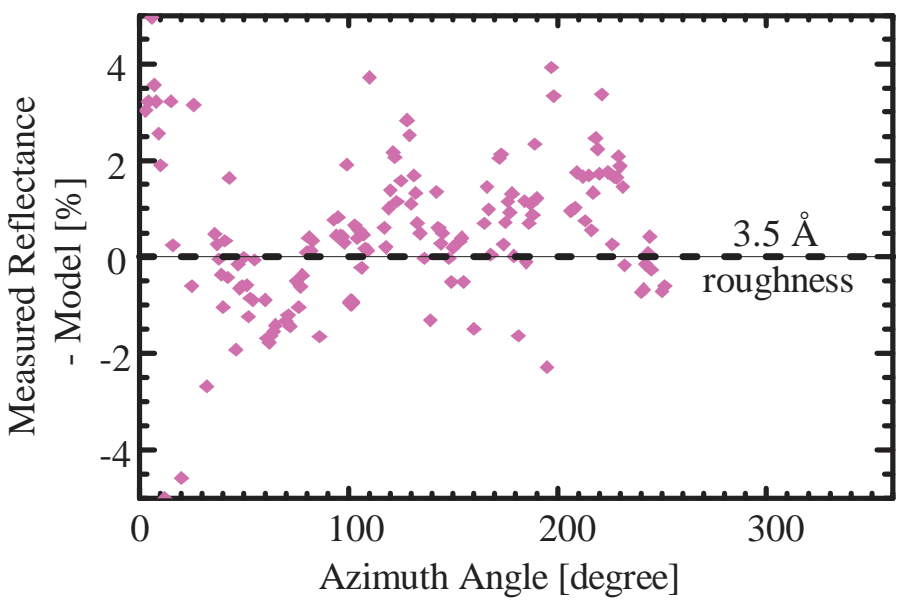

Figure 10: The difference between the measured reflectivity (c.f., Equation 2) and theoretical reflectance model for a prototype optic with $\mathrm{W} / \mathrm{SiC}$ multilayers is plotted versus azimuth angle. The systematic errors associated with these data are estimated to be $\sim 1-2 \%$, and the statistical errors are negligible. performance measurements, these measurements were performed every degree as previously described, and there is negligible background involved in these measurements. In addition, the normalization of the throughput measurements is much better than the performance measurements, and they are much quicker to perform since scanning through angle space is not necessary.

As previously mentioned, the mirrors were all coated with $\mathrm{W} / \mathrm{SiC}$ multilayers. At $8 \mathrm{keV}$, the combined reflectivity of the upper and lower mirrors is relatively high at $83 \%$ and is not strongly dependent upon the exact multilayer coating including the interfacial microroughness. Thus, the $8 \mathrm{keV}$ X-ray measurements can be used to calibrate out the axial throughput factor $\varepsilon_{\text {figure }}$ (which is independent of energy) in order to extract the measured reflectivity at $40 \mathrm{keV}$ :

$$
\mathrm{R}_{\text {measured }}(40 \mathrm{keV}, \sigma, \alpha)=\mathrm{T}_{\text {measured }}(40 \mathrm{keV})\left\{\mathrm{R}_{\text {model }}(8 \mathrm{keV}, \sigma, \alpha) / \mathrm{T}_{\text {measured }}(8 \mathrm{keV})\right\},
$$

The difference between the measured and model reflectivity at $40 \mathrm{keV}$ with $3.5 \AA$ surface roughness is plotted in Figure 10, which illustrates a good fit to the measured data. Note that all theoretical multilayer reflectivities quoted in this paper have been calculated using the IMD 4.1.1 software created by D.L. Windt. ${ }^{7}$

\subsection{Pt/SiC multilayer reflectivity}

The W/SiC multilayers have good response up to $70 \mathrm{keV}$, but are limited above this point by the $70 \mathrm{keV} \mathrm{W}$ absorption edge. As previously described in Section 2, the NuSTAR design calls for $\mathrm{Pt} / \mathrm{SiC}$ multilayers for the inner $2 / 3$ of the telescope while the $\mathrm{W} / \mathrm{SiC}$ multilayers will only be used for the outer layers where the graze angle is large enough that the reflectivity above $70 \mathrm{keV}$ would be minimal even with $\mathrm{Pt} / \mathrm{SiC}$ multilayers. Measurements of the $\mathrm{Pt} / \mathrm{SiC}$ multilayer response demonstrate that excellent interface widths of $3 \AA$ can be achieved for $\mathrm{Pt} / \mathrm{SiC}$ multilayers of the NuSTAR design. The measured $\mathrm{Pt} / \mathrm{SiC}$ response for graze angles up to $5^{\circ}$ is plotted in Figure 11. The measured data is in good agreement with the theoretical response predicted for $\mathrm{Pt} / \mathrm{SiC}$ multilayers with $3 \AA$ surface roughness and exceeds the NuSTAR reflectance requirement. For comparison 3,4 and $5 \AA$ roughness models are also plotted.

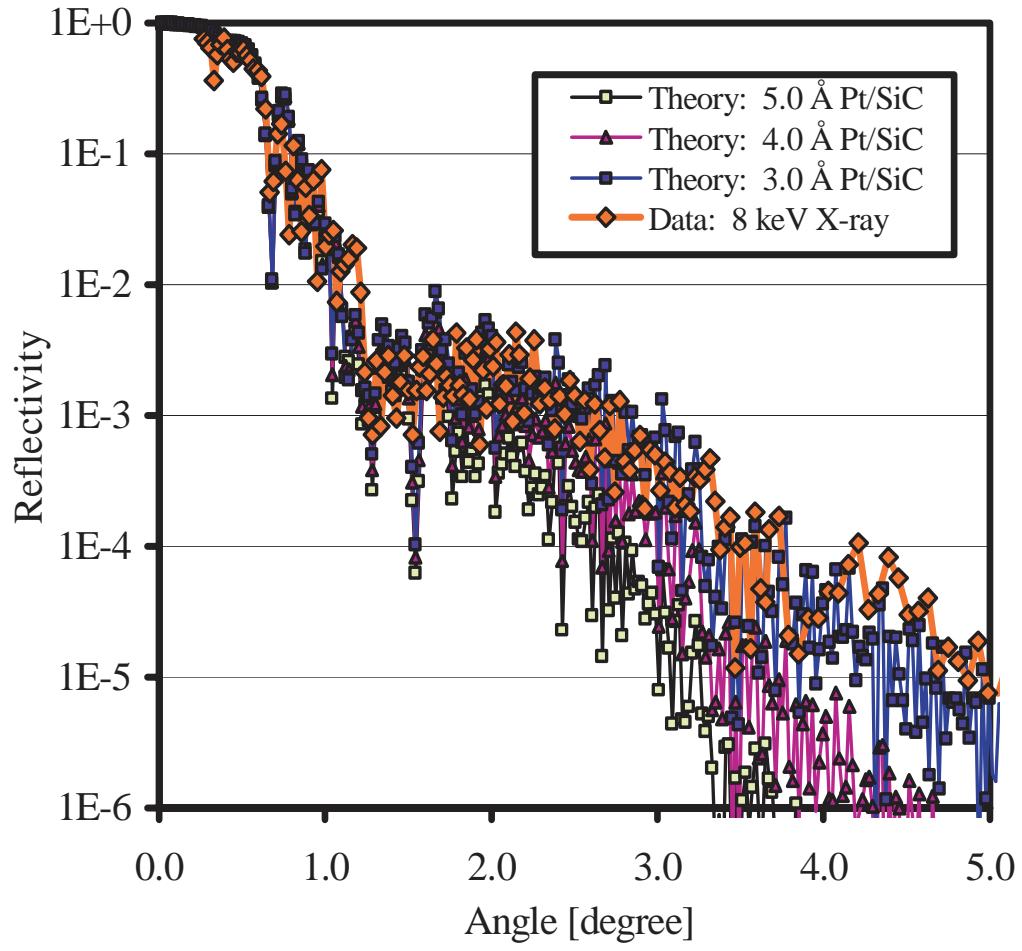

Figure 11: $\mathrm{Pt} / \mathrm{SiC}$ reflectivity data are plotted versus graze angle. For comparison 3, 4 and $5 \AA$ roughness models are also plotted, and the data is shown to be consistent with the $3 \AA$ model. 


\section{SUMMARY}

This paper has outlined a clear path to leverage the experience we have gained through the HEFT balloon mission into the successful development of optics for the NuSTAR satellite mission. A summary of the key aspects that will assure this success is detailed in Table 2.
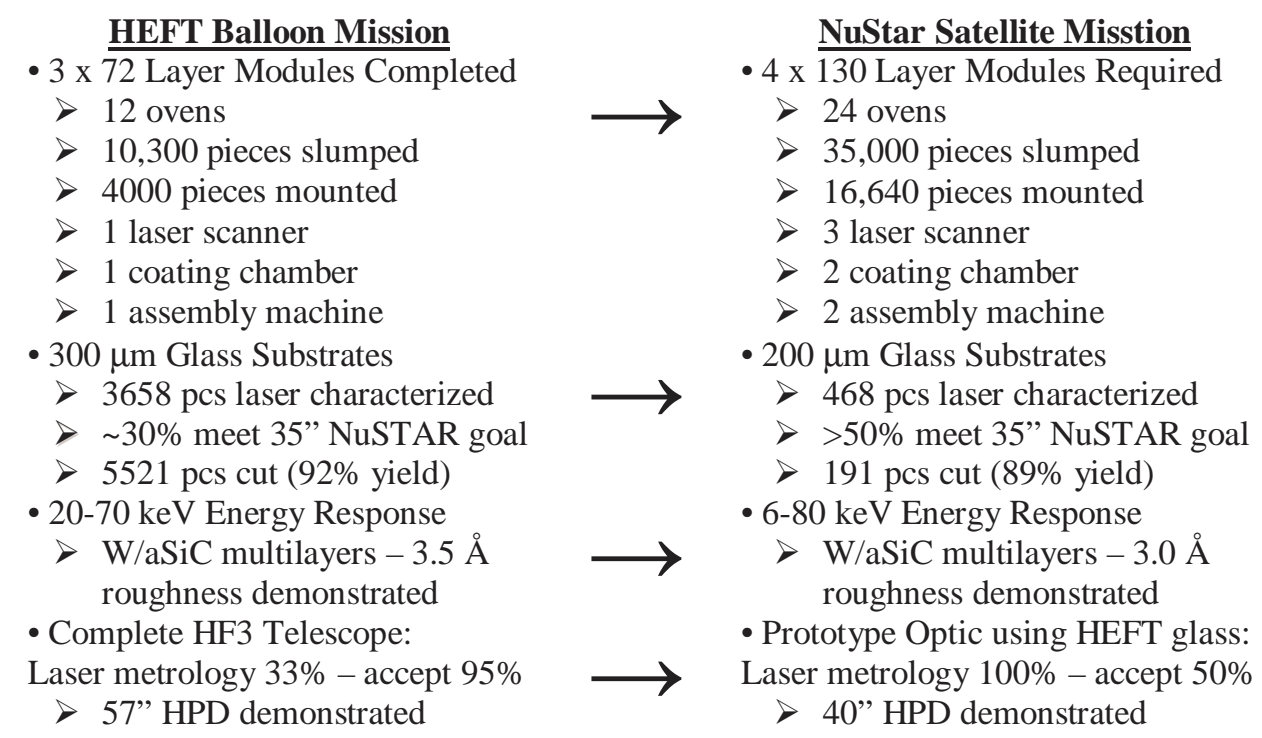

Table 2: Summary of key aspects in transferring the experience gained from HEFT into the success for NuSTAR.

\section{ACKNOWLEDGEMENTS}

Special Thanks to the staff at ESRF for their assistance with the high-energy measurements. This work is supported by a NASA grant to Columbia University: NAG5-5260 (Thermally Formed Optics for X-Ray and Gamma-Ray Astronomy), and by NASA grants to the California Institute of Technology with sub-awards to Columbia: Caltech No. 1019776 (High Energy Focusing Telescope) and Caltech No. 1046806 (Critical Technologies for the Constellation Hard X-Ray Telescope).

\section{REFERENCES}

${ }^{1}$ F.A. Harrison, W.W. Craig, et al., Proc. SPIE 5488-12, 2004.

2 J.E. Koglin, F.E. Christensen, J. Chonko, W.W. Craig, T.A. Decker, C.J. Hailey, F.A. Harrison, C.P. Jensen, M. Sileo, D.L. Windt and H. Yu, " Development and Production of Hard X-ray Multilayer Optics for HEFT," Proc. SPIE 4851 (2003) 6007-618.

${ }^{3}$ J.E. Koglin, H.C. Chen, F.E. Christensen, J. Chonko, W.W. Craig, T.R. Decker, M.A.Jimenez-Garate, C.J. Hailey, F.A. Harrison, C.P. Jensen, M. Sileo, D.L. Windt and H. Yu, "Development of Precision Hard X-ray Multilayer Optics with Sub-Arcminute Performance," Proc. SPIE 4851 (2003) 673-683.

${ }^{4}$ J.E. Koglin, C.M.H. Chen, F.E. Christensen, J. Chonko, W.W. Craig, T.R. Decker, K. S. Gunderson, C.J. Hailey, F.A. Harrison, C.P. Jensen, K.M. Madsen, M. Stern, D.L. Windt and H. Yu, E. Ziegler, "Production and calibration of the first HEFT hard X-ray optics module", Proc. SPIE 5168 (2004) 100-111.

${ }^{5}$ M. Jimenez-Garate, W. Craig, C. Hailey, F. Christensen, and A. Hussain Opt. Eng. 39(11), p. 2982, 2000.

${ }^{6}$ C. J. Hailey, F. E. Christensen, W. W. Craig, F. A. Harrison, J. E. Koglin, R. Petre, W. W. Zhang, "Fabrication and Performance of Constellation-X Hard X-ray Telescope Prototype Optics Using Segmented Glass", Proc. SPIE 5168 (2003) 90-99.

${ }^{7}$ D.L. Windt, "IMD - Software for modeling the optical properties of multilayer films", Comput. Phys. 12, 360 (1998) 